\title{
Attitude as an Expressible Info-Operational Reaction to a Perceived/Purposed Object/Objective
}

\author{
Research Article
}

Volume 1 Issue 1- 2020

\begin{abstract}
Author Details
Florin Gaiseanu*

Science and Technology of Information, Bucharest (Romania) and Barcelona (Spain)

*Corresponding author

Florin Gaiseanu, Science and Technology of Information, Bucharest (Romania) and Barcelona (Spain)
\end{abstract}

Article History

Received: December 07, 2020 Accepted: December 15, 2020 Published: December 17, 2020

\begin{abstract}
The definition of the psychological concept of attitude is still a controversial problem, and the scholars have not yet reached a consensus on this important issue. The attitude is defined in various ways, as a mental and emotional "construct" not directly detectable, or as a "psychological tendency expressed by evaluating a particular entity". However, as attitude is an achievement of the mind during the exploration of reality, it is naturally to approach first of all the nature of mind and the relation with the cognition processes from the informational perspective. Therefore, in this paper it is investigated the concept of attitude from a completely new point of view, starting from the informational nature of consciousness. It is shown that the informational structure of consciousness can be fully described by the activity of seven distinct cognitive centers and the attitude can be defined actually as an informational reactive output with respect to an object/objective either perceived or mentally proposed. The attitude is thus the result of a decisional info-processing of an input internal or external information, expressible by the specific informational center managed by the brain associated with this activity, defined suggestively as Iwant. It is shown that attitude is consequently a function of all other six centers, which intervene in the decisional process as decisional criteria or as priority contributing components, and these centers can become dominant or inactive. In agreement with some previous studies and with the neuro-connections of specific regions of the brain, it is shown that emotions contribute to attitude, but also the personal state, the inherited predispositions, the social interactions, the life experience and the trust in the objective, if this is a proposed project. Associated with the attitude, behavior is different, depending on all cognitive centers.

Keywords: Attitude, Informational systems, Neuro-connections, Cognitive centers, Operational reaction, Perceived/Purposed object/ objective, Decision/Judgment, Attitude/Behavior definitions
\end{abstract}

\begin{abstract}
Abbreviations: CDC: Center of Decision and Command; IES: Info-Emotional System; MIS: Maintenance Informational System; GTS: Genetic Transmission System; IGG: Info-Genetic Generator; IC: Info-Connection
\end{abstract}

\section{Introduction}

Attitude is one of the main property of the human psyche, which expresses a reaction within the interaction with the surroundings and the body itself, therefore is an attribute of the mind. The longtime research-history on this issue highlights the practical relevance of this property in psychology, sociology, political science and in other connecting disciplines [1]. The correlation of mind with the body is a very old problem, probably even since the first beginnings of the human existence, expressed by philosophy [2] and continued by psychology, neuroscience and behavior sciences [3], but remained not well understood and defined yet. Since the first decades of the last century it was recognized the importance and the large range of applications of the attitude concept [4], but the definition of this concept is still under discussion. According to the recent studies, showing that the attitude is related with a category of problems concerning "how individuals perceived the world" and how they "behave", the definition of the attitude should refer to "people, groups, ideas, and objects", which mainly express whether individuals "like or dislike them" [1], and the main meaning of this concept is articulated on cognitive, affective and behavioral aspects $[1,5]$.

Within such studies, little attention was however paid to the brain neuro-implications in the investigation of attitude as a response of the nervous system, but it was however recognized that attitude might be related to the neuro-activities beyond of amygdala, which is the alarm component of the brain included in the emotional limbic system, and not only limited to it [6]. The necessity of a deep understanding of the functions and of the operability of the brain, of the mind and of the associated cognitive activities, has engaged in such a multidisciplinary scientific issue not only neurology [7,8] and the neuroscience [9], 
stimulated by the non-invasive and nondestructive novel techniques of experimental investigation, driven especially by the spectacularly development of the microelectronic/microtechnology science and engineering, but also the information science [10-12].

According to the psychological studies, the attitude is a mental and emotional "hypothetical construct" which characterizes the human personality, a concept which "cannot be observed directly, only be inferred from people's actions" [3], or "a psychological tendency" to express the evaluation of a particular quality and the degree of "favor or disfavor" granted to it [13]. Such descriptive/narrative solutions to define the attitude, as part of a large category of "vague" definitions [14] limited to particular examples, was a consequence of the lack of suitable models and significant results allowing to understand the nature and architecture of consciousness, which were not available till now. A convergence concerning a fine definition of the attitude was therefore not achieved yet. The intervention of the information science in the field of brain and the associated properties allowed a successful advance on this domain [15-17]. In this paper it is defined and discussed from the informational perspective the concept of attitude with respect to an object/objective perceived during the exploration of reality or mentally proposed, on the basis of the informational structure of consciousness.

\section{Analysis of the Informational Structure of Consciousness Defining the Attitude}

The above definitions of attitude can be only synthetized in one word: information. And the following citation "although in some sense we do infer a person's attitude from what says or does, it would be a mistake to assume that for this reason attitudes are not real" [3], could be also be resumed as information. Such descriptive definitions were necessary because of the lack of introduction of a powerful and indispensable tool of investigation: information. According to some recent studies, information is part of our world and a component of everything in our surroundings. This is a result of the concurrence of more than one branch of science, i,e. philosophy [18], physics [10] and more recently the information science [17]. Matter itself is a form including information and energy, because the microparticle components include ("embody") information and energy of structuration [19]. The human being [11], as also the living structures in general [20], need and dispose of more information than trivial matter, both to survive and to work in an animating way.

Approaching from the informational perspective consciousness and human organism, we may discover that human is a bipolar information-matter entity, connected to the informational field/data of the environment and of the body itself: to maintain the metabolic processes the body need to be supplied with matter (foods, air, water), eliminating matter-wastes and information to adapt its functions and behavioral reactions for survival. Analyzing consciousness from this perspective, we have to observe that this is also a "concept" which cannot be observed directly, only by means of the actions and consequences. We have therefore to look deeper into the structural properties of consciousness and the cognition properties, in order to determine in a scientific manner what means actually attitude. For this, we have to observe that the informational contact with reality is possible by means of the sensors, receiving and transmitting the perceived information to the brain, where this information is stored under various forms (Figure 1 left side): (i) as momentary, short-time memorized information in the prefrontal cortex, serving as the perception and orientation in the surroundings; (ii) as longtime memorized information, stored especially in the hippocampus structure of the brain; (iii) as abilities, like driving a bike or a car, which is a more complex info-motor acquired information, stored especially in cerebellum. Therefore, a center of acquirement and storing of information (CASI) could be defined, supported by the sum of all regions of the brain specified above. As memory includes entire our life experience, it is specifically reflected in consciousness as a cognitive center, which can suggestively defined by Iknow, as shown in the right side of the Figure 1. These definitions help us to understand better the organization of the brain functions, classified in distinct informational categories, according to their specific attributions.

An immediate and direct function related to memory refers to the operational attributes of the brain, consisting basically in judgment of the received information, according to the personal accepted/verified/ learned criteria. Indeed, something can be classified as good or bad, accepted (YES) or rejected (NO) according to a reference value, which is a judgment criterion. The operational unit YES/NO is a decision in a binary (Bit) system, so information. The info-operational part of the brain supporting and managing the decisions can be defined as a center of decision and command (CDC), represented in the left side of Figure 1, connected especially with the cortex hemispheres. The main execution elements are basically related with the vocal system and muscles, allowing the immediate expression of the decision. The decision is expressible by language, and/or by gesture, facial mimic, corporal posture and movements, which represent actually the "corporal language". The decisional operability is detected in consciousness by the cognitive center, suggestively named Iwant, which is actually the informational output of the informational system of the human body. We have to observe therefore that human receives information by means of input (sensorial) channels storing it in Iknow and returns information by an info-output channel Iwant, which is actually the attitude.

However, a full decision cannot be only the result of the "cold" judgment. As the above cited definition stipulates [3], the attitude might include also an emotional component. Such a component is expressed by the activity of the info-emotional system (IES) of the human organism, supported in the brain mainly by the limbic structures, operating by thalamus, hypothalamus, midbrain and amygdala, which is the alarm component (Figure 1 left side), and is reflected in consciousness by the cognitive center Ilove, expressing the associative relation with the life and survival needs (Figure 1 left side).

The material structure of the human organism is automatically managed by the maintenance informational system (MIS), connected mainly to the hypothalamus, brain stem and medulla in the brain, and with the digestive system in the body, which supports the metabolic processes, reflected in consciousness by the cognitive center Iam, as it is shown in Figure 1 in the left and right side respectively. The respiratory system is closely involved in this function. The center Iam expresses actually the self "image" and own perception in the conscious mind, characterizing the personal vitality, health, power and corporal features and virtues. The automatic management is completed by the functions of the genetic transmission system (GTS) and info-genetic generator (IGG) (Figure 1 left side), responsible for the sexual activity of the body and for the body development according to the age respectively, connected with hypophysis and hypothalamus in the brain and with the reproduction organs in the body. These systems are also reflected in consciousness by the cognitive centers Icreate and Icreated (Figure 1 right side). The Info-Connection (IC) represented in the left side of Figure 1 operates in the cingulate cortex the selection YES/NO of the received information before to be sent to the conscious frontal cortex by means of the anterior cingulate cortex [21], or manages the preliminary information from the external to the internal world, by means of the posterior cingulate cortex for the infofiltration according to the internal criteria and beliefs [22], reflected in consciousness by the cognitive center Ibelieve.

As in an operational info-system, the nature of CDC decisions depends not only on the nature, quality and quantity of the input information stored in the memory and the criteria of operation, but also on the information acquired and inherited by means of the genetic channels (IGG), and also on the informational signals received by the internal sensors connected to MIS and GTS, expressing the self-status of the body and the associated needs. All these signals could ask a certain priority of operations, intervening/interfering in/with the decisional 
process. IC is also an active center, assuring the selection of a certain information according to individual favorable criteria, or with the priority order. Therefore, although the attitude is mainly operated by CDC, perceived and expressed by Iwant, all other info-systems contribute to the decision and thus to attitude, as it is discussed in detail below.

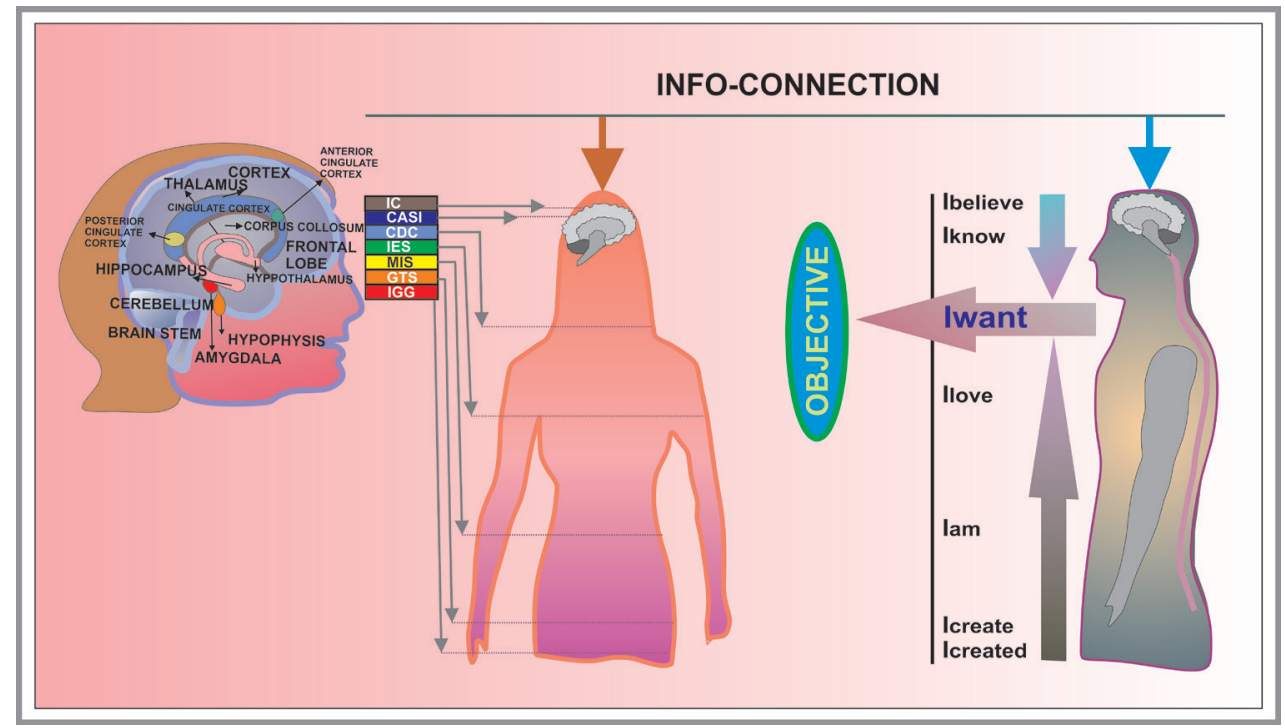

Figure 1: Schematic representation of the components of the informational system of the human body (left side) and of the cognitive centers of consciousness (right side), revealing the attitude expressed by Iwant with respect to a certain objective.

\section{Attitude as a reactive Informational Expression and the Associate Characteristic Properties}

The attitude can be defined only with respect to a certain object or objective, as it is shown in Fig. 1. When the attention is focused on a certain objective, either mental or external object/situation perceived during the exploration of the surrounding reality, the attitude refers to the personal reaction on this. Although the same information is available for more than one person, each participant connected to the same reality/information perceives and evaluates/judges it by means of own criteria, so the attitude with respect to it is different. The attitude is therefore personal and is a consequence of the operability of own informational system and available information.

The evaluation Good/Bad or Accepted/Rejected of an information resulted from the external reality or from mental perception, is closely related to the life experience accumulated in memory (Iknow). Therefore, the operability of CDC (Iwant) is closely related with CASI (Iknow), because CDC must search and select from CASI the necessary information for a pertinent judgment/evaluation and compare it with the internal reference standards and norms of conduction, learned during the childhood from parents and school, or accumulated by own experience during the life. The evaluation process is therefore dependent on the Good/Bad experience with respect to similar type of objects/objectives and the associated criteria. The emotional experience (Ilove) is also accumulated in memory (Iknow) and can be detected during the remembrances recall. The emotions are triggered by information itself and are associated to them. The negative emotional experiences will become a rejection criterion for new similar experiences, so the center Ilove plays a determinant role in the decision, sometimes a decisive one, in spite of the importance of other judicious criteria. The contribution of the activity of these cognitive centers to the decisional process is marked in Figure 1 right side by an arrow oriented to the center Iwant.

The inherited predispositions, abilities, inclinations, tendencies and preferences manifested by the center Icreated could play a decisive role in the decisional process, engaging the center Icreated as a priority player in decision making [23]. Traditions, religion of the parents and/ or own religion, specific culture of the childhood realms and home habits, education during the first years of life, part of the Icreated experience is a major and stable support for the decisional process sometimes for the entire life $[23,24]$. Often, such inherited or adopted principles during the childhood reflected by Icreated are the primary support for the transmission of this informational baggage to the new generation during the educational process by means of Icreate. From this point of view, the center Ibelieve depends closely on the center Icreated, because it reflect the most consolidated convictions validated by generations, taken from parents, by education, or validated by own experience during the experimental existential practice.

The center Icreate is also very important in the decisional process, active especially during the period of formation of own family and the education of the new generation. This center is a priority when the objective is family and its maintenance, so could interact with the other centers on the social or economical relations with the other persons, institutions, organisms or the society in general. Icreate has therefore a social dimension, intervening in the relations with others, during the professional or any other interactions related to the economical and financial resources. On the other hand, these interactions influence the self-image and status reflected in the center Iam, depending on the successful or unsuccessful results during or after these interactions.

The personal status, concerning the health and power necessary to comply a purposed objective, is also an important decisional criterion, so the attitude with respect to such objective depends also on the cognitive center Iam. The operational GTS is a strong competitor in the decisional making depending on the proposed objective and therefore Icreate intervene also in the design and generation of the attitude. The contribution of the automatic components are shown in Fig. 1 by the corresponding upwards oriented arrow to the center Iwant. The contemplation of the personal capability for a long-time process necessary to comply a certain difficult objective, asking full engagement and force, like in sportive competitions or other competition tasks, is a judicious attitude before the itself engagement.

The dynamics of attitude depends therefore by objective, time and the activity of all cognitive centers of mind. If we define the attitude with respect to the objective $(\mathrm{O})$ by the symbol Ao, this is a function (f) on time $(\mathrm{t})$ and on the activity of the centers Ibelieve, Iknow, Ilove, Iam, Icreate, Icreated, so Ao can be written as:

$\mathrm{Ao}=\mathrm{f}($ Ibelieve, Iknow, Ilove, Iam, Icreate, Icreated, $\mathrm{t})(1)$

This definition shows that attitude is actually expressed by the activity of the center Iwant, as it is represented in Fig.1 right side by the arrow oriented to the objective. The contribution of each component is distinct and depends on the nature of the objective and on the time. 
The attitude concerning a perceived object is rather contemplative/ evaluative and expressible by the bipolar YES/NO reaction, whereas the attitude referred to a purposed objective is engaging, mobilizing the personal recourses of attention, perseverance and power of achievement (through Iam). Each of cognitive center. i.e. the center Iknow (experience on the proposed issue), Ilove (the emotional attachment to the proposed objective), Icreate (social experience and interactions), Icreated (predispositions and inherited abilities) and Ibelieve (the stable trust in the successful objective) plays a significant role in successful results. One or more cognitive centers could be dominants with respect to an objective in certain moment, or be inactive. Each of these cognitive centers is educable by the power of the centers Iwant and Ibelieve, maintaining the will force on the right direction, by exercises and practice. Such a stable preoccupation is welcome to comply objectives concerning the carrier, professional preparation, or any other particular objective, including the personal physic or physique development.

The evaluative attitude is specifically referred to the perceived objects, allowing their rapid/spontaneous evaluation, although could operate also with respect to a purposed objective. The first impression is a momentary "flash", but the attitude concerning objectives related to planning are longer-time dependent. The impulsive/instinctive attitude is a characteristic attribute of species, derived from adaptation/ survival requirements. Indeed, the amygdala is a specialized component of the limbic system warning on the potentially dangerous situations, developed on the evolutionary scale just to comply with such a function. The associated emotional cognitive center Ilove is spontaneously connected during such an evaluation process, transmitting thus to the center Iwant to accept (YES) or to reject (NO) the perceived characteristics of the objective. The center Ibelieve supplies also the experiential acquirements allowing to select the suitable YES/NO decision. The judgment/evaluation criteria, validated as reference values and stably memorized in Iknow and related with the activity of Icreate, Icreated and Iam, are used also during this rapid evaluation. These reference values refers to the social interactions (Icreate), educational/inherited mentality concepts (Icreated) or personal characteristics (Iam). The global evaluation expressible by such evaluative attitude is therefore favorable or unfavorable (YES or NOT), depending on each of these factors, in concordance with the earlier defined characteristics describing attitude as "a psychological tendency that is expressed by evaluating a particular entity with some degree of favor or disfavor." [13].

Attitude is often associated with behavior [25-27], because attitude is visible/detectable by the external signals like facial expression, gestures, posture, typical reactions, interpretable also as behavior. However, this could be really different of attitude, especially taking into account some circumstantial situations, when the real attitude, as a personal decisional evaluation or reaction with respect to the focused object/objective cannot be really expressed, because of public/ social limitative norms or personal motives of limitation. Behavior Bo with respect to an object/objective or situation can be therefore defined by the general relation:

$\mathrm{Bo}=\mathrm{f}($ Ibelieve, Iknow, Iwant, Ilove, Iam, Icreate, Icreated, t) (2)

where the activity of the cognitive center of personal (intimate) decision Iwant is one of the participating components to the behavioral manifestation, but not the determinant one. As in the case of attitude, the participating level of each component is of course different, depending on the nature of the objective and also on the time.

The attitude is personal and depends on the properties of own perception and filtering informational system according to its operational capability, personal criteria and life experience. That is because the attitude was defined as a function of own cognitive centers, which refers to the self ("I"). The reactivity is referred to the position with respect to the object/objective of the perception or mentally proposed, from the perspective of each of these center, globally applied. The attitude is operational, because intervene in own consciousness and could be stored in memory (Iknow) as a new experience, intervening in own consciousness experiential field or in the external world. The first momentary intervention is expressed by vocal reaction, mimics, gesture/posture, triggered especially by emotions, as a primary effect. Emotions are closely related therefore with the motor system and are a form of rapid reactive operability for adaptation. The more suggestive argument is that of the neuroconnection of amygdala, which is the alarm component of the emotional system. The cited definition of attitude, showing that "we do infer a person's attitude from what he or she says or does" [3], refers just to the visible/detectable external operability expressed by attitude. The result of decision can be self-monitored also, tacitly saved in the internal storing "library" (Iknow), without external manifestation, and can be used latter within a new experiential info-operative judgment/ evaluation. Attitude can be defined actually in general terms as an automatic or volitional/decisional reactive response of the human organism to internal or external purposes, either by contemplative/ evaluating or engaging motivation.

According to earlier cited study [3], "attitudes are learned, people are not born with attitudes", but this definition would refer probably to attitude taking into account only the conscious expression, learned from parents or acquired during the interaction within own community. However, the reaction expressible by attitude is a born (instinctive) property, independently on the way and the form of which it is expressed, because human is a reactive organism, as well as other living systems, starting from monocellular to more complex multicellular entities [20]. The living systems and perhaps especially human, are reactive informational systems, which respond to informational stimuli by a reaction, so by an attitude with respect to respective stimuli. This is an intrinsic characteristic feature of the living systems. When a child cries of hungry, he express by this attitude a native need impulse. The genetic inherited information is manifested in a specific and distinctive personal way. The native reactions expressed by attitudes refers not only to the species (genetic) acquirements, but also to the additional individual transgenerational acquirements obtained by epigenetic mechanisms during the life of the parents $[28,29]$. The specificity is a result of the distinctive reactive properties of individuals.

\section{Conclusion}

Attitude is a result of the informational interaction with the environment or to own body. As an attribute of mind, the attitude was defined taking into account the structure of the informational system of the human body and the associated distinctive and specific cognitive centers of consciousness, allowing a fine tuning of the cognitive functions and associated properties. It was shown that attitude is mainly expressible by the cognitive center suggestively defined as Iwant, either if the attitude is manifested in the external environment by vocal, facial mimic, gesture or corporal posture and action, or deliberately stored in memory in a tacit way for latter utilization. The cognitive center Iwant expresses a decision and thus an attitude with respect to an object/objective, either after perception or mental proposition. Iwant is one of the seven contributing cognitive centers of consciousness, among Iknow (memory), Ilove (emotional reaction), Iam (self-status), Icreate (social inter-relations), Icreated (inherited predispositions), Ibelieve (selective trust information) and is the results of the global or partial participation of these centers, depending on time and on the nature of the objective. Such definition is more precise than the narrative/descriptive definition, because it is focused and based on the nature of attitude concept as a reactive response to information.

The attitude is therefore a reactive info-operational process, expressible as a reaction to the environmental/inner stimuli received/proposed 
as an input information, and represents actually an output of the informational system of the organisms for adaptation and survival. The attitude can be expressed or not, and is memorized in own library of data, acting as experiential reference for latter decisional processes. The attitude is expressible as an evaluation process especially of a perceived object/situation, or a contemplating/self-engaging operational process, when it is referred to a proposed mental objective. The attitude could be learned during the life experience, but also is manifested as instinctive inherited/acquired impulses of species, or accumulated during the formational/educational process from parents during the first years of childhood.

The behavior is a concept similar with attitude but not identical, expressible also by the contribution of the cognitive centers of consciousness, including this time the center Iwant as a component and not as the determinant contributor, taking into account that sometimes in certain circumstances the sincere attitude cannot be expressible directly.

\section{Acknowledgment}

To my son Adrian Gaiseanu, eminent specialist, son and father, and to his family. To my daughter Ana-Maria Gaiseanu, highly following the best prerogatives in family, life and profession, and to her family. To Traian and Monica and to their family.

To corresponding neuropsychology and information science forums and scientists, to Romanian Academy.

Grateful thanks to the Managing Editor and to this Journal for kind invitation to participate with this contributing paper.

\section{Conflict of Interests}

Author has no conflicts of interest.

\section{References}

1. Wolf Lukas J, Haddock Geoffrey, Maio Gregory R. Attitudes, Oxford Research Encyclopedia of Psychology, Oxford University Press; 2020: 211-216.

2. Gaiseanu Florin. Language Patterns and Cognitive-Sentient Reality: Certainty/Uncertainty in Cognitive-Sentient Exploration of Reality, Chapter in Media Models to Foster Collective Human Coherence in the PSYCHecology. Stephen Brock Schafer (eds), USA; 2019: 49-72.

3. Perloff Richard M. The Dynamics of Persuasion: Communication and Attitudes in the Twenty-First Century, In: Lawrence Erlbaum Associates (Edn), Mahwah: New Jersey London; 2020.

4. Strauss Anselm (1945) The Concept of Attitude in Social Psychology. Rhe Journal of Psychology 19(2): 329-339.

5. Morris Jerry W, Stuckhardt Michael H (1977) Art Attitude: Conceptualization and Implication. Studies in Art Education 19(1):2128.

6. Cunningham William A, Haas Ingrid J, Jahn Andrew. Attitudes. In: J. Decety, JT Cacioppo (eds.) The Oxford Handbook of Social Neuroscience. New York: Oxford University Press; 2011.

7. Gaiseanu Florin (2019) Informational Neuro-Connections of the Brain with the Body Supporting the Informational Model of Consciousness. Archives in Neurology \& Neuroscience 4(1): 1-6.

8. Gaiseanu Florin (2020) Information Based Hierarchical Brain Organization/Evolution from the Perspective of the Informational Model of Consciousness. Archives in Neurology \& Neuroscience 7(5): $1-9$.

9. Gaiseanu Florin (2019) Informational Mode of the Brain Operation and Consciousness as an Informational Related System. Archives in Biomedical Engineering \& Biotechnology 1(5): 1-7.

10. Gaiseanu Florin (2016) Consciousness as Informational System of the Human Body. Consciousness and Life Physics, Cosmology and Astrophysics Journal 16(1): 14-25.
11. Gaiseanu Florin (2019) The Informational Model of Consciousness: Mechanisms of Embodiment/Disembodiment of Information. NeuroQuantology 17(4): 1-17.

12. Gaiseanu Florin (2019) Human/Humanity, Consciousness and Universe: Informational Relation. NeuroQuantology 17(5): 60-70.

13. Eagly Alice H, Shelly Chaiken (1998) Attitude, Structure and Function. Handbook of Social Psychology. In: DT Gilbert, Susan T Fisk, \& G Lindsey (eds); New York: McGowan-Hill; 269-322.

14. Altmann Tanya (2008) Attitude: A Concept Analysis. Nursing Forum 43(3):144-50.

15. Gaiseanu Florin (2020) Information-Matter Bipolarity of the Human Organism and Its Fundamental Circuits: From Philosophy to Physics/ Neurosciences-Based Modeling. Philosophy Study 10(2): 107-118.

16. Gaiseanu Florin (2019) Information: from Philosophic to Physics Concepts for Informational Modeling of Consciousness. Philosophy Study 8(8): 368-382.

17. Gaiseanu Florin (2018) Informational Model of Consciousness: From Philosophic Concepts to an Information Science of Consciousness. Philosophy Study 9(4): 181-196.

18. Draganescu Mihai (1990) Information of Matter (Informatia materieii in Romanian). Bucuresti: Editura Academiei Romane.

19. Gaiseanu Florin (2020) Physics of Consciousness and Life: Informational Model of Consciousness - Information in Neurosciences, Biocomputers and Biosystems (Fizica Constiintei si a Vietii: Modelul Informational al Constiintei - Informatia in Neurostiinte, Biocomputere si Biosisteme (in Romanian); GlobeEdit (OmniScriptum International Group, Germany).

20. Gaiseanu Florin (2020) What Is Life: An Informational Model of the Living Structures. Biochemistry and Molecular Biology 5(2): 18-28.

21. Gaiseanu Florin (2020) Informationally Assisted Mind-Body Equilibrium and Health: Specific ACC Contribution from the Perspective of the Informational Model of Consciousness. EC Psychology and Psychiatry J 9(5): 37-49.

22. Gaiseanu Florin (2020) Info-Relational Cognitive Operability of the Posterior Cingulate Cortex According to the Informational Model of Consciousness. International Journal of Psychological and Brain Sciences 5(4): 61-68.

23. Gaiseanu Florin (2018) Destiny or Free Will Decision? A Life Overview from the Perspective of an Informational Modeling of Consciousness Part II: Attitude and Decision Criteria, Free Will and Destiny. Gerontology \& Geriatric Studies 4(1): 354-360.

24. Gaiseanu Florin (2019) Destiny or Free Will Decision? A Life Overview from the Perspective of an Informational Modeling of Consciousness Part I: Information, Consciousness and Life Cycle. Gerontology \& Geriatric Studies 4(1): 400-405.

25. Amherst Icek Ajzen, Fishbein Martin (1977) Psychological, AttitudeBehavior Relations: A Theoretical Analysis and Review of Empirical Research. Bulletin 84(5): 888-918.

26. Andrich David, Styles Irene M (1998) The Structural Relationship Between Attitude and Behavior Statements From the Unfolding Perspective. American Psychological Association 3(4): 454-469.

27. Marcinkowski Tom, Reid Ala (2019) Reviews of research on the attitudebehavior relationship and their implications for future environmental education research. Environmental Education Research 25(4)L 459-471.

28. Gaiseanu Florin (2019) Epigenetic Information-Body Interaction and Information-Assisted Evolution from the Perspective of the Informational Model of Consciousness. Archives in Biomedical Engineering \& Biotechnology 2(2): 1-6.

29. Gaiseanu Florin (2019) The Silent Voice of Those Who are no Longer: Transgenerational Transmission of Information from the Perspective of the Informational Model of Consciousness. Gerontology \& Geriatric Studies 5(1): 482-488. 